\title{
An ICT Supported Holistic Approach for Qualitative and Quantitative Energy Efficiency Evaluation in Manufacturing Company
}

\author{
Hendro Wicaksono ${ }^{1, *}$, Kiril Aleksandrov ${ }^{1}$, Sven Rogalski $^{2}$, and Jivka Ovtcharova ${ }^{2}$ \\ ${ }^{1}$ Intelligent Systems and Production Engineering/ Process \\ and Data Management in Engineering FZI Research Center for Information Technology, \\ Karlsruhe, Germany \\ \{wicaksono, aleksandrov\}@fzi.de \\ ${ }^{2}$ Institute for Information Management in Engineering, Karlsruhe Institute of Technology, \\ Karlsruhe, Germany \\ \{sven.rogalski, jivka.ovtcharova\}@kit.edu
}

\begin{abstract}
The global climate change and the rising of energy prices force manufacturing companies to regulate their energy usage. A suitable step to achieve that is the introduction of energy management. This paper presents an ICT based holistic approach to help manufacturing companies in the implementation of energy management system. It consists of methods to support quantitative and qualitative energy efficiency evaluation of their operations. The approach uses an ontological knowledge base containing the structures and rules representing best practices as reference of energy efficiency to support the qualitative evaluation. In the approach, we also develop measurement figures called Energy Performance Indices (EPI) to determine the energy efficiency degrees in different organizational parts of the company. The paper also describes the application of the approach in a small medium sized manufacturer.
\end{abstract}

\section{Introduction}

The global climate change and the rapid growth of industrialization, have led to a significant increase of energy demand that results to constantly increasing of electricity, gas, and oil prices. Meanwhile, the changes of social, technical, and economic conditions in the market have challenged manufacturers to deal with the requirements for various and complex products. Hence, energy efficiency in accordance with the economization of production costs is an important competitive factor in the energyintensive industry. A common solution to achieve this is through a corporate energy management. Energy management defines the sum of all processes and measures to ensure minimal energy consumption by a given demand including the implementation of organization, information structure, and tools [1]. The standard ISO 50001 describes the requirements for energy management systems in industrial companies.

\footnotetext{
* Corresponding author.

C. Emmanouilidis, M. Taisch, D. Kiritsis (Eds.): APMS 2012, Part I, IFIP AICT 397, pp. 25-32, 2013.

(C) IFIP International Federation for Information Processing 2013
} 
However, most of the manufacturing companies face problems in implementing the energy management standards, due to the distributed and unstructured energy related information within the company. The information is difficult to be accessed by all stakeholders in the company. Best practices to avoid energy wasting are only known by some employees. This is due to the knowledge gap and insufficient access methods to the knowledge.

To overcome this problem, this paper introduces an approach utilizing information and communication technology that supports a holistic evaluation of energy efficiency both qualitatively and quantitatively. Qualitative evaluation focuses on the criteria that can be observed but not measured using figures, such as energy wasting situations, or energy efficient best practices. The qualitative evaluation is performed by employing a knowledge base, which is accessible by all of personals and systems in the company. The best practices and knowledge about energy efficient and energy wasting activities are standardized, formalized and stored in the knowledge base. Researchers have been applying ontologies as knowledge representation in various domains to solve the shared understanding problems among people and even software. In this paper, the knowledge base is represented with OWL (Web Ontology Language $)^{1}$ combined with SWRL ${ }^{2}$. Quantitative evaluation evaluates energy efficiency using measurement figures. Therefore it is possible to determine the energy efficiency degree in different organizational parts or processes in company. The figure is called Energy Performance Indices (EPI). In this paper, energy performance indicators that are able to measure the energy efficiency in different level of company organizations, is proposed.

\section{Literature Overview}

Researchers have developed knowledge based approach since end of 80s to manage information from different sources to support the decision making, in order to improve the efficiency, flexibility, and reliability of manufacturing processes [2][3]. Knowledge and communication models were developed to relate different aspects in manufacturing and to allow coordination of different components in the manufacturing organization [4] [5]. For the last ten years, researchers have been applying ontologies as knowledge representation in various domains, including manufacturing. Ontology has been used as a solution to the shared understanding problems among people and even software [6]. It has also been proven to harmonize the knowledge gap between customers and manufacturers during the requirement elicitation phase [7] and to solve the semantic ambiguities [8]. Different approaches to create ontologies in manufacturing domain were developed to model the processes, product, and resources as well as their relation in production [9] [10]. In energy management domain, to improve the energy efficiency especially in households, analysis of energy consumption of different appliances from various manufacturers and technologies is performed with the help of ontology as the integration model [11] [12]. Furthermore,

\footnotetext{
http: //www.w3.org/TR/2004/REC-owl-ref-20040210/

http: / /www.daml.org/rules/proposal/
} 
ontology combined with rules is also used to represent the knowledge base of an intelligent energy management system that monitors and controls the energy consumption in a household [13]. Until now, there is still a lack of knowledge based approach to support the evaluation tasks of energy management in manufacturing. This paper introduces a novel method using ontology and rules represented knowledge to formalize and to structure the knowledge and the best practices as references to regulate the energy consumption in manufacturing.

By using metrics as the performance indicators, an assessment for the state and condition of complex systems is easier and faster, due to their simple representations. The figures to measure energy efficiency are classified into different categories, such as absolute, relative, classification, and relational figures [14]. This paper focuses on relational figures, since they describe the energy consumption based on cause-effect relation, for instance, energy consumption per a produced product piece. German Engineer Association (VDI) introduces different technical figures related to energy evaluation [15]. The energy requirements relative to the amount of products depend very much on the type and quality of the products. An approach to fix this problem is by converting the output product values into a particular unit [16]. Some metric systems have been developed to evaluate the energy efficiency for entire industry sectors, such as EPI from Energy Star and ODEX [17] [18] [19]. However, there is still lack of figures that are able to measure energy efficiency of different organization levels in a single manufacturing company, such as of a machine, of a production line, and of the whole factory. This paper proposes a method that addresses this problem.

\section{Overview of the Solution}

In this paper, we propose an ICT framework that supports the implementation of energy management in manufacturing companies consisting of both tools for quantitative and qualitative energy efficiency evaluation. Fig. 1 illustrates the overview of the framework. The information about products, production processes, and resources are collected from different IT systems of the company, such as ERP, MES (Manufacturing Execution System). The energy related data are gathered from Energy Monitoring and Data Acquisition (EMDA) System. Those different data are aggregated and analyzed, in order to have the relation between products, production processes, and resources e.g., machines, surrounding factors (i.e., temperatures), and energy consumptions.

The data analysis to create the relations could be done semi-automatically with the help of algorithms, such as data mining. Rules representing the energy usage patterns are deduced from the data mining result. The rules allow the identification of energy usage anomalies or inefficiencies. Knowledge engineer of the company could also models the common best practices of energy management with rules. These rules are stored in the form of a knowledge base as a reference of energy efficiency qualitative evaluations. The collected data are used to assign the values of the mathematical model that calculates the EPI. The simulation and optimization module is responsible to help the production planners to perform production planning that consider not only the costs but also energy efficiency. The module contains an optimization algorithm for finding the optimal production schedule that takes the rules as the constraints and the maximum EPI as the objective function. 


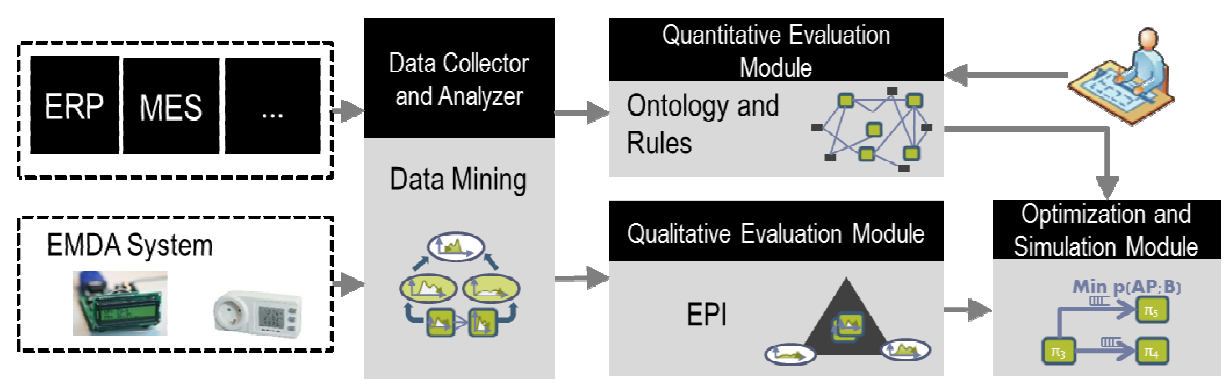

Fig. 1. Overview of the approach

\section{Qualitative Evaluation using Knowledge Base}

In our work, we develop OWL ontology for manufacturing energy management. There exists PLM ontology that models the structure and relationships among products, processes, and resources [9] [20]. In our work, we consider knowledge elements representing the energy management with an additional related knowledge, such as ancillary, transport or intra-logistics and energy conversion facilities. Equation (1) gives an example of a SWRL rule that models energy wasting practice in a stainless steel manufacturer. It describes, if an oven is active during a heat treatment process and the heating system is located in the same zone is still turned on, then it is considered as an energy inefficiency condition. The rule is created manually, since it represents the common knowledge.

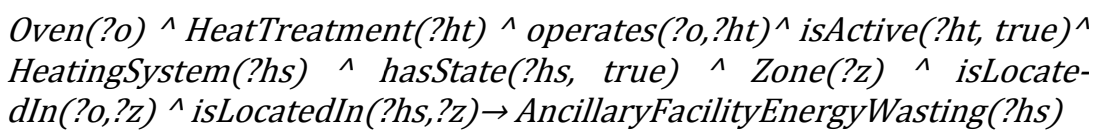

Fig. 2 illustrates a decision tree resulted from the data mining that extracts the relation between product properties and the energy consumption in forging process. The tree represents the common and normal situation that the forging process of products, having material type M1 and outer diameter greater than $115 \mathrm{~cm}$, consumes energy between 175 and $268 \mathrm{~kW}$. The tree is then transformed into SWRL rules, for example into rule (2), to detect the energy inefficiency or anomaly states. The rule implies, if the forging process on presser machine consumes energy more than $268 \mathrm{~kW}$, then there is something wrong in the process causing energy inefficiency.

Product(?p)^ hasMaterialType(?p, “M1") ^ hasOuterDimeter(?p,?od) ^
swrlb:greaterThan(?od,115) ^ Presser300oT(?ps) ^ Forging (?f) ^ op-
erates(?ps,?f) ^ produces(?o,?p) ^ hasEnergyConsumption(?ps,?e)
swrlb:greaterThan(?ps,268) $\rightarrow$ ProductionFacilityEnergyWasting(?ps)

By using the knowledge, the energy wasting can be detected, hence energy efficiency can be evaluated. By assigning the variables with current states in the company, running a rule engine on the SWRL rules and using $\mathrm{SQWRL}^{3}$ to query the knowledge base as shown in (3), the inferred information can be retrieved from

3 http://protege.cim3.net/cgi-bin/wiki.pl?SQWRL 


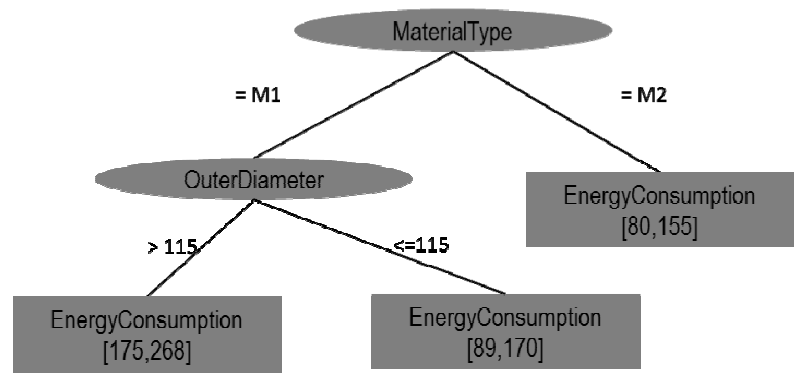

Fig. 2. Decision tree of data mining result

the rules. The retrieved information can be further processed by using the company's messaging or alerting system.

$$
\begin{aligned}
& \text { AncillaryFacilityEnergyWasting(?h) } \rightarrow \text { sqwrl:select(?h) } \\
& \text { ProductionFacilityEnergyWasting (?h) } \rightarrow \text { sqwrl:select(?h) }
\end{aligned}
$$

\section{Energy Performance Indices (EPI) for the Quantitative Evaluation}

To develop energy efficiency metric for a single manufacturing company, first, it is important to define the system boundaries and a model that covers the different organization levels of production. In our work we develop UPNT, which is based on UPN model [21], and considers the machine, production line and factory level. The basic elements of the UPNT model are energy conversion (U), production (P), ancillary $(\mathrm{N})$, and transport $(\mathrm{T})$ facilities. The production and transport facilities are modeled as subclasses of ontological class ManufacturingResource, the energy conversion as EnergySource, and ancillary facility as BuildingElement in ontology. Fig. 3 illustrates the boundaries of different manufacturing organization levels. It can be seen, that the inputs of a production facilities are made up from direct and an indirect ones. The direct inputs are from utility companies or conversion facilities. The indirect inputs are provided due to the fact that the production facility receives the outputs of ancillary and transportation facility. Therefore the energy cost of a production facility is also ascribed with the output of the connected ancillary and transport facilities. Equation (2) calculates the EPI for factory level. The calculations for the machine and production line levels are also performed similarly.

$$
E P I_{l}=\frac{V_{l}}{\sum_{k}^{p} P_{k}+\sum_{i=1}^{m} \alpha_{i} A_{i}+\sum_{j=1}^{n} T_{j}+\sum_{v}^{q} A_{v}}
$$

$$
\text { where } \begin{array}{lll}
\mathrm{V}_{1} & = & \text { output value generated by production line } l[€] \\
\mathrm{P}_{\mathrm{k}} & = & \text { input of production facility } k[€] \\
\alpha_{\mathrm{i}} & = & \text { ratio of use input ancillary facility }=[0,1] \\
\mathrm{A}_{\mathrm{i}} & = & \text { input ancillary facility } i \text { to each production facility }[€] \\
\mathrm{T}_{\mathrm{j}} & = & \text { used transport facility } j[€] \\
\mathrm{A}_{\mathrm{v}} & = & \text { input ancillary facility } v \text { for the whole production line }[€]
\end{array}
$$




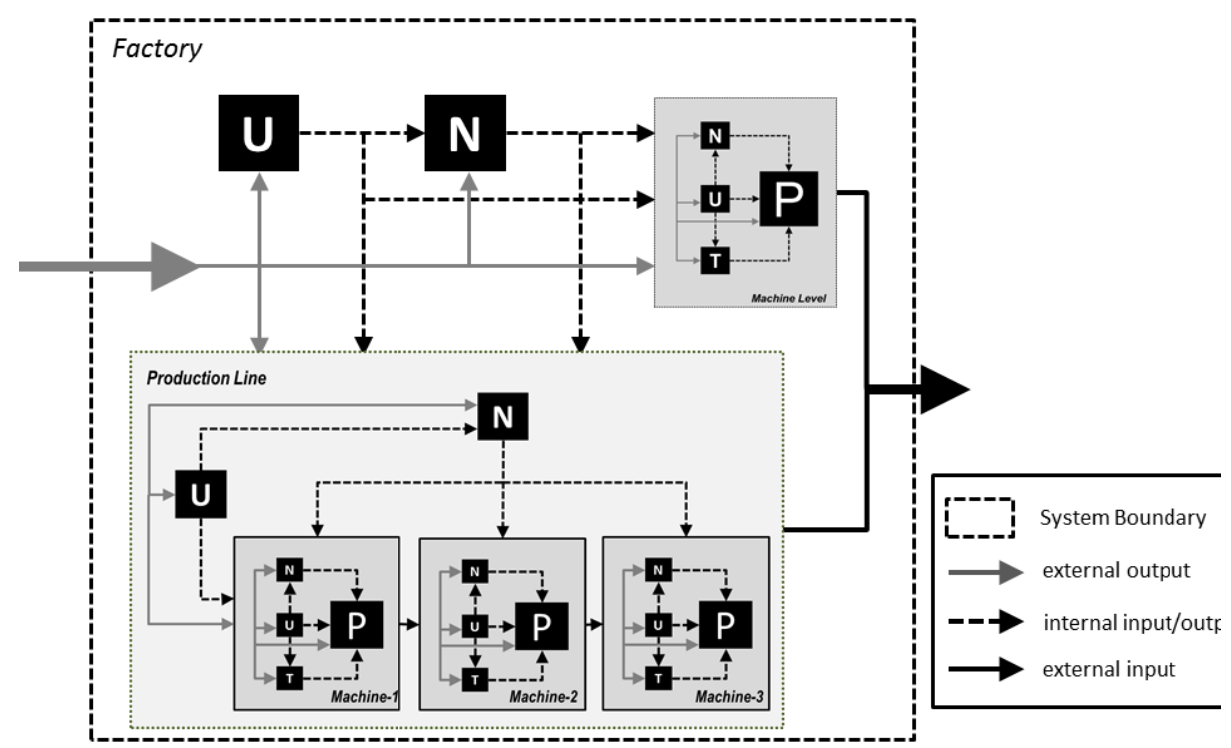

Fig. 3. Energy flows and system boundaries for EPI calculation

The calculation method is applied in a stainless steel manufacturer. Table 1 shows examples of the calculation results in machine and production line levels. Theoretically, the EPI values should be positive rational numbers. Less than one value means that energy costs exceed the supply and therefore should not occur. Otherwise, it would be an alarm signal, because the process is extremely inefficient. Higher values are conceivable when a process generates a lot of values and /or require very little energy. A value greater than one does not automatically make a positive contribution margin, as there are other costs in addition to the energy costs that must be met. However, this is not the objective of the EPIs. Rather, they should provide a comparison of different alternatives in the choice of equipment to be used.

Table 1. EPI calculation results in stainless steel production

\begin{tabular}{ll}
\hline Description & EPI \\
\hline M: Oven & $21,0-30,3-49,6$ \\
M: Presser (1x) and Drop hammer (2x) & $11,4-21,0-21,9$ \\
M: Turning machine (3x) & $10,5-20,9-32,6$ \\
M: Boring Machine, CNC machine (1x) & $15,7-17,7$ \\
M: Ring roller(2x) & $15,5-18,9$ \\
L1: Oven (EPI = 21,0), Drop hammer (EPI = 21,9), Ring- & 19,5 \\
rolling (EPI = 15,5) & \\
L2: Oven (EPI = 49,6), Presser (EPI = 11,4), Ring-roller & 19,3 \\
(EPI = 18,9), Rolling- machine (EPI = 21,7) & \\
L3: Oven (EPI = 30, 3), Drop hammer (EPI = 21,0), Rolling & 21,3 \\
machine (EPI = 20,9), & \\
\hline
\end{tabular}




\section{Summary}

This paper presents an approach that employs ICT method to support the holistic evaluation of energy efficiency in the manufacturing on both quantitative and qualitative ways. The knowledge base provides the structured and formalized knowledge as references of best practices in the company related to energy efficiency. By using the data mining, the previously unknown relations among products, manufacturing processes, facilities, resources and energy consumption are revealed and incorporated into knowledge base. The ontology represented knowledge base provides formalized knowledge that allows common understanding among employees and software in the company. By using developed EPI, the energy consumption's performance of different production facilities and production lines is measured quantitatively. Therefore, it helps the managers and production planners to plan their production not only by considering the costs, but also by considering the energy efficiency. Manufacturers can benefit from this approach by receiving an evaluation tool that enables them, despite the ever-intensifying competition with higher energy costs and scarce natural resource and an increase of production of complexity, to compete with competitors. The approach also improves the awareness of the stakeholders to make decision by strongly taking considerations whether their decision affects the energy efficiency in the company.

\section{References}

1. Kahlenborn, W., Kabisch, S., Klein, J., Richter, I., Schürmann, S.: DIN EN 16001: Energy Management Systems in Practice - A Guide for Companies and Organisations. In: Bundesministerium für Umwelt, Naturschutz und Reaktorsicherheit, Berlin, Germany (2010)

2. Hsu, C., Skevington, C.: Integration of data and knowledge in manufacturing enterprises: A conceptual framework. Journal of Manufacturing Systems 6(4), 277-285 (1987)

3. Weber, D., Moodie, C.: A knowledge-based system for information management in an automated and integrated manufacturing system. Robotics and Computer-Integrated Manufacturing 4(3-4), 601-617 (1988)

4. Guerra-Zubiaga, D., Young, R.: A manufacturing model to enable knowledge maintenance in decision support systems. Journal of Manufacturing Systems 25(22), 122-136 (2006)

5. Oztemel, E., Tekez, E.: Integrating manufacturing systems through knowledge exchange protocols within an agent-based Knowledge Network. Robotics and Computer-Integrated Manufacturing 25(1), 235-245 (2009)

6. Noy, N., McGuinness, D.: Ontology Development 101: A Guide to Creating Your First Ontology (2001), http://www.ksl.stanford.edu/people/dlm/papers / ontology101/ontology101-noy-mcguinness.html (accessed on July 10, 2012)

7. Wicaksono, H., Schubert, V., Rogalski, S., Ait Laydi, Y., Ovtcharova, J.: Ontology-driven Requirements Elicitation in Product Configuration Systems. In: Enabling Manufacturing Competitiveness and Economic Sustainability, pp. 63-67. Springer, Heidelberg (2012)

8. Valiente, M.C., Garcia-Barriocanal, E., Sicilia, M.A.: Applying an ontology approach to IT service management for business-IT integration. In: Knowledge-Based Systems, vol. 28, pp. 76-87. Elsevier Science Publishers, Amsterdam (2012) 
9. Lemaignan, S., Siadat, A., Dantan, J.Y., Semenenko, A.: MASON: A Proposal For An Ontology of Manufacturing Domain. In: Proc. Workshop on Distributed Intelligent Systems: Collective Intelligence and Its Applications, pp. 195-200. IEEE Computer Society, Washington (2006)

10. Panetto, H., Dassisti, M., Tursi, A.: ONTO-PDM: Product-driven ONTOlogy for Product Data Management interoperability within manufacturing process environment. Advanced Engineering Informatics (26), 334-348 (2012)

11. Shah, N., Chao, K.-M., Zlamaniec, T., Matei, A.: Ontology for Home Energy Management Domain. In: Cherifi, H., Zain, J.M., El-Qawasmeh, E. (eds.) DICTAP 2011 Part II. CCIS, vol. 167, pp. 337-347. Springer, Heidelberg (2011)

12. Rossello-Busquet, A., Brewka, L., Soler, J., Dittmann, L.: OWL Ontologies and SWRL Rules Applied to Energy Management. In: Proc. 13th International Conference on Modelling and Simulation, pp. 446-450. IEEE Computer Society, Washington DC (2011)

13. Wicaksono, H., Rogalski, S., Kusnady, E.: Knowledge-based Intelligent Energy Management Using Building Automation System. In: Proceeding of the 9th International Power and Energy Conference, pp. 1140-1145. IEEE Computer Society, Washington (2010)

14. Kals, J.: Betriebliches Energiemanagement - Eine Einführung. Verlag W. Kohlhammer, Stuttgart (2010)

15. Verein Deutscher Ingenieure (VDI): Energetic characteristics: definitions - terms - methodology. Beuth Verlag GmbH, Düsseldorf (2003)

16. Müller, E., Engelmann, J., Löffler, T., Strauch, J.: Energieeffiziente Fabriken planen und betreiben. Springer (2009)

17. Boyd, G., Zhang, G.: Measuring Improvement in the Energy Performance of the U.S. Cement Industry. In: Technical Report, Duke University, Durham, North Carolina (2011)

18. European Energy Efficiency: Analysis of ODYSSEE indicators. In: Technical Report. Department of Energy \& Climate Change, London (2012)

19. Tanaka, K.: Assessing Measures of Energy Efficiency Performance and their Application in Industry. In: Technical Report, International Energy Agency (IEA), Paris, France (2008)

20. Raza, M.B., Harrison, R.: Ontological Knowledge Based System for Product, Process and Resource Relationships in Automotive Industry. In: Proc. International Workshop on Ontology and Semantic Web for Manufacturing, Heraklion, Crete, Greece (2011)

21. Fünfgeld, C.: Energiekosten im Betrieb. Solar Promotion GmbH-Verlag, Munich (2000) 